\title{
S4Net: Single stage salient-instance segmentation
}

\author{
Ruochen Fan ${ }^{1}$, Ming-Ming Cheng ${ }^{2}$, Qibin $\mathrm{Hou}^{2}$, Tai-Jiang $\mathrm{Mu}^{1}$, Jingdong $\mathrm{Wang}^{3}$, Shi-Min $\mathbf{H u}^{1}(\varangle)$ \\ (C) The Author(s) 2020.
}

Abstract In this paper, we consider salient instance segmentation. As well as producing bounding boxes, our network also outputs high-quality instance-level segments as initial selections to indicate the regions of interest. Taking into account the category-independent property of each target, we design a single stage salient instance segmentation framework, with a novel segmentation branch. Our new branch regards not only local context inside each detection window but also the surrounding context, enabling us to distinguish instances in the same scope even with partial occlusion. Our network is end-to-end trainable and is fast (running at $40 \mathrm{fps}$ for images with resolution $320 \times 320)$. We evaluate our approach on a publicly available benchmark and show that it outperforms alternative solutions. We also provide a thorough analysis of our design choices to help readers better understand the function of each part of our network. Source code can be found at https://github.com/RuochenFan/S4Net.

Keywords salient-instance segmentation; salient object detection; single stage; region-of-interest masking

\section{Introduction}

Rather than recognizing all objects in a scene, humans only care about a small set of interesting instances of objects [1]. A recent experiment [2] has demonstrated that interesting objects are often visually salient,

* An earlier version of this paper was presented in IEEE CVPR 2019.

1 BNRist, Tsinghua University, Beijing 100086, China. Email: R. Fan, frc16@mails.tsinghua.edu.cn; T.-J. Mu, mmmutj@gmail.com; S.-M. Hu, shimin@tsinghua.edu.cn (囚).

2 Nankai University, Tianjin 300071, China. E-mail: M.-M. Cheng, cmm@nankai.edu.cn; Q. Hou, andrewhoux@gmail.com.

3 MSRA, Beijing 100086, China. E-mail: jingdw@microsoft.com.

Manuscript received: 2019-09-16; accepted: 2020-04-12 reflecting the importance of detecting salient objects. Locating objects of interest is also essential for a wide range of computer graphics and computer vision applications. Such a capability underpins many modern applications (e.g., image manipulation and editing [3-5] and robotic perception [6]), providing initial regions that might be of interest to users or robots for image editing or scene understanding. Salient object detection aims to identify the most humanly visually distinctive objects in an image [7]. Ground truth for saliency detection can be provided by a saliency map annotated by one or more humans. Free viewing is the most common method for saliency modeling, in which participants view an image for a fixed duration and salient regions are inferred from their eye movements [8]. A good salient object detection algorithm should be able to output a predicted saliency map in good agreement with a ground truth saliency map. As in Ref. [9], we are also interested in detecting salient instances given an input image. Salient instance segmentation is similar to salient object detection, in that it aims to detect the most distinctive objects in a scene, but differs in that it also identifies each individual instance, i.e., outputting an accurate segment for each instance and assigning it a unique label (see Fig. 1).

Cognitive psychology $[10,11]$ and neurobiology [12] suggest that human cortical cells may be hard wired to preferentially respond to high contrast stimuli, i.e., feature separation between foreground and background regions plays a central role in salient object perception [13, 14]. Effectively modeling foreground-background separation using local and global contrast [15, 16], background priors [17], and Gaussian mixture color models (as in GrabCut [18]), etc., has proven useful in a variety of traditional salient-object and figure-ground segmentation tasks. Recently, convolutional neural networks (CNNs) 


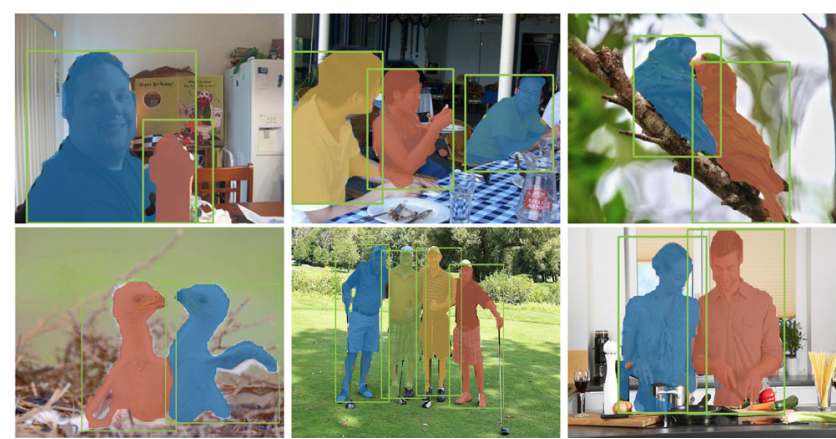

Fig. 1 Example salient instances output by our approach, which detects and segments them regardless of their semantic category. Each category-agnostic salient instance is highlighted with a unique color Our results provide well-segmented candidates of interest to users, significantly reducing human efforts needed for image manipulation.

have become the dominant method for nearly all closely related tasks, e.g., salient object detection [19-21], semantic instance segmentation [22-24], and generic object detection [25-27]. While these CNNbased methods have achieved remarkable success by learning powerful multi-level feature representations for capturing different abstract appearance variations in the target categories, they often ignore the important ability to separate target objects from their background.

Existing CNN-based instance segmentation methods use either RoIPooling [28, 29], RoIWarp [30], or RoIAlign [31] to capture feature information inside bounding boxes. In contrast, we propose a region feature extraction layer, RoIMasking, to explicitly incorporate foreground-background separation, to improve salient instance segmentation. As in the figure-ground segmentation method GrabCut [18], we explicitly mark the region surrounding the object proposal as the initial background, and explore foreground-background feature separation for salient instance segmentation in our segmentation branch. More specifically, we flip the signs of the feature values surrounding the proposals. The RoIMasking layer based segmentation branch is then integrated with the efficient single-stage object detector FPN [32], to pixel-wise detect the segment of each salient instance. Interestingly, our RoIMasking scheme is quantizationfree and scale-preserving, allowing detailed information to be successfully detected. Furthermore, our model is end-to-end trainable and runs at $40 \mathrm{fps}$ on a single GPU when processing $320 \times 320$ images.

To verification the utility of our method, we apply our salient instance detector to the popular weakly-supervised semantic segmentation task. As in Refs. [33, 34], we use the detected salient instances on the tiny ImageNet dataset $[34,35]$ as heuristics to train various well known semantic segmentation networks. We evaluate the results on the popular PASCAL VOC 2012 semantic segmentation benchmark [36] and show that our results outperform by a large margin state-of-theartmethods $[33,34]$ that leverage traditional salient object cues $[16,19]$.

To sum up, the contributions of this paper are:

- an end-to-end single-shot salient instance segmentation framework, which achieves stateof-the-art, real time, performance;

- a new RoIMasking layer which models feature separation between target objects and their surrounding background to provide high-quality segmentation.

\section{Related works}

Salient instance segmentation is a relatively new task. Seminal methods were recently proposed by Zheng et al. [37] for finding salient objects at bounding box level. However, this method misses the important segmentation information, which is essential for applications such as image editing [3, 5] and weakly supervised segmentation [33]. Li et al. [9] formally define the salient instance segmentation problem as jointly identifying salient regions as well as individual object instances. They also proposed an MSRNet [9] framework for this task. However, it was excessively reliant on the quality of precomputed edge maps (e.g., using MCG [38]) and produced sub-optimal results for complicated real-world scenes (see also Section 4). Salient instance segmentation is closely related to three major computer vision tasks: salient object detection, object detection, and semantic instance segmentation.

\subsection{Salient object detection}

Salient object detection aims to both detect the most distinctive objects in a given scene and segment them from it. Early salient object detection methods typically depend on global or local contrast cues $[15,16,39,40]$. They designed various hand-crafted features (e.g., color histograms and textures) for each region [41-43] and fused these features in either a manually-designed [15] or learned [44] manner. Because of their weak ability to preserve the integrity 


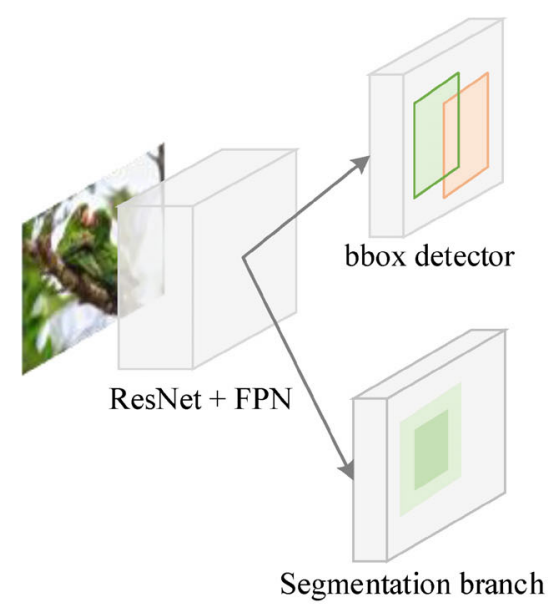

(a) Framework

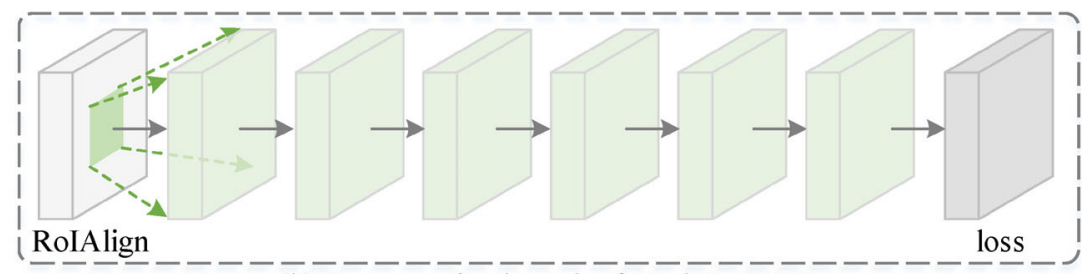

(b) Segmentation branch of Mask R-CNN

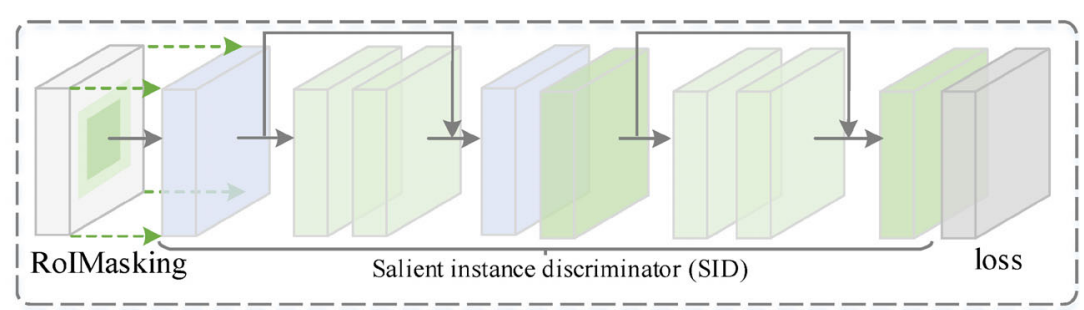

(c) Segmentation branch of our method

conv dilated conv pooling

loss

Fig. 2 Pipeline of the proposed method. (a) Simplified framework. For simplicity, we omit the backbone used. See Ref. [32] for more information. (b) Segmentation branch proposed in Mask R-CNN [31], comprising a stack of consecutive convolutional layers. (c) Our proposed segmentation branch which further enlarges the size of the receptive field but has the same number parameters as in (b).

of salient instances and the lack of robustness of hand-crafted features, these methods were gradually replaced by CNN-based data-driven methods $[9,19$ 21, 45-47]. The key issues in using salient object detection methods for salient instance segmentation task are two-fold. Firstly, it is difficult to preserve the integrity of the salient objects, because the distinctive regions may be parts of the interesting instances. Secondly, salient object detection is a binary problem and hence unsuitable for instance-level segmentation.

\subsection{Object detection}

The goal of object detection is to produce all bounding boxes for objects in various semantic categories. Earlier work mostly relied on handengineered features (e.g., SIFT [48], SURF [49], and HOG [50]). Different types of image pyramids were used to leverage information across scales. Recently, the emergence of CNNs has greatly promoted the development of object detectors. For example, RCNN [25] and OverFeat [51] regard CNNs as sliding window detectors for extracting high-level semantic information. Given a stack of precomputed proposals $[52,53]$, these methods compute feature vectors for each proposal using CNNs and then feed the features into a classifier. Later work [28, 29] took entire images as inputs and applied region-based detectors to feature maps, substantially improving speed. Faster R-CNN [26] broke through the limitation of using precomputed proposals by introducing region proposal networks (RPN) into CNNs. In this way, a whole network could be trained end-to-end, offering a better trade-off between accuracy and speed compared to previous work. However, all the above methods aim to output reliable object bounding boxes rather than instance segments.

\subsection{Semantic instance segmentation}

Earlier semantic instance segmentation methods [22-24, 54] are mostly based on segment proposals generated by segmentation methods [38, 52, 55]. In Ref. [30], Dai et al. produced segmentations by leveraging a multi-stage cascade to gradually refine rectangle regions from bounding box proposals. Li et al. [56] proposed integrating a segment proposal network into an object detection network. More recently, Ren et al. implemented a Mask R-CNN framework, extending the Faster R-CNN [26] architecture by introducing a segmentation branch. While significant results, these methods are unsuitable for our task for two reasons. Firstly, not all categories and objects are salient. Secondly, the semantic instances all belong to a predefined collection of categories, and these methods lack the important ability to deal with unknown categories, i.e., class-agnostic salient instances.

\section{S4Net}

The design choices of our method are based on the application requirements: high-quality salient 
instance segmentation in real time. Our end-to-end single-shot salient instance segmentation framework, S4Net, is built on top of the state-of-the-artsingle-shot object detector for efficiency.

\subsection{Observation}

Recent instance-level semantic segmentation methods $[31,56]$ have shown a strong ability to segment semantic instances, using RoIWarp [30], or RoIAlign [31]. However, the segmentation branches of these methods only focus on features inside proposals to describe appearance variation within target instances, and lack the ability to distinguish different instances.

Before CNN-based methods became popular, utilizing feature separation between foreground and background was the dominant mechanism used for similar tasks such as salient object detection [15-17] and figure-ground segmentation [18]. The ability to effectively model foreground-background feature separation is so powerful that these methods $[15,17,18]$ could achieve remarkable success by utilizing such feature separation in the target image alone, without any additional information provided by training images. An example is shown in Fig. 3. Users only need to draw a rectangle region (shown in red) around the target object. The GrabCut method [18] uses foreground/background color models, in the form of Gaussian mixture models (GMM). Notice in

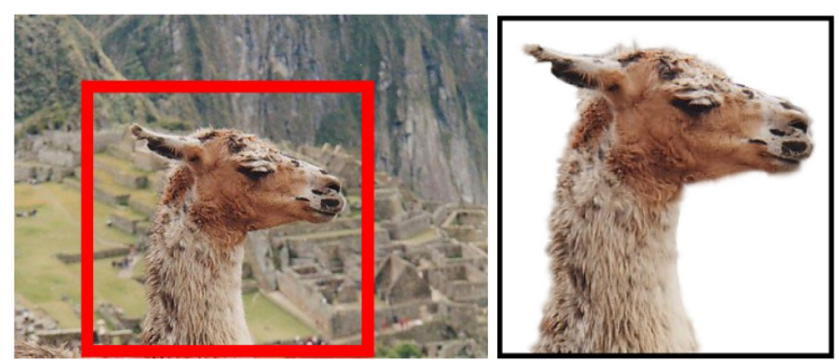

Fig. 3 Interactive figure-ground segmentation using GrabCut [18] this example that parts of the target object have very similar colour to parts of the background. However, the GMM color model effectively captures the slight color differences (indistinguishable to human eyes).

Unfortunately, the ability to take advantage of such powerful foreground-background feature separation is lacking in existing CNN-based segmentation methods. Motivated by this observation, we aim to explicitly leverage more features corresponding to the background area, to help make the salient instances more prominent, as shown in Fig. 5. This scheme allows more features representing the background area (relative to the salient instance) to be considered by the segmentation branch, enlarging the receptive field of the segmentation branch and meanwhile enhancing the contrast between foreground and background, especially when there is occlusion.

\subsection{Framework}

The pipeline of S4Net is shown in Fig. 2; it has two components: a bounding box detector and a segmentation branch. Both components share the same base model. As in most object detection works, we use ResNet-50 [57] as our base model.

\subsubsection{Single-shot object detector}

For efficiency of the entire network, we adopt a singleshot object detector [58] with FPN [32] as the base model, to leverage its multi-level features. To reduce the runtime, we discard the lateral connections to conv2 while keeping the rest unchanged (i.e., conv3conv6). Four detection heads are connected to each lateral layer as shown in Fig. 2(a). The head structure is the same as in Faster R-CNN [26], but with different strides to perform detection at multiple scales.

\subsubsection{Single-shot segmentation branch}

Unlike existing instance level semantic segmentation methods such as Mask R-CNN [31], our segmentation

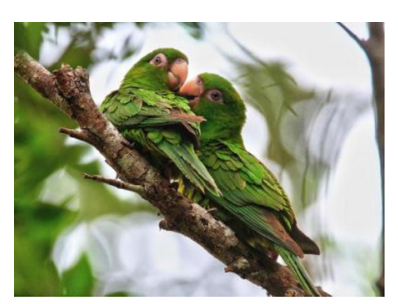

(a) Input image

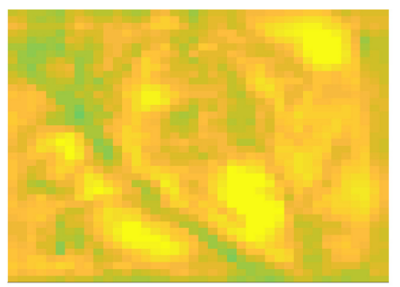

(b) Feature map before masking

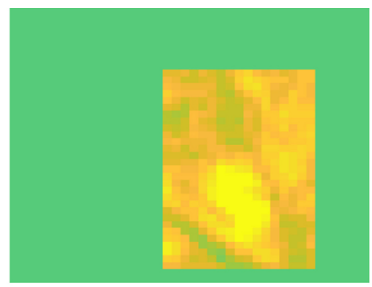

(c) Binary RoIMasking

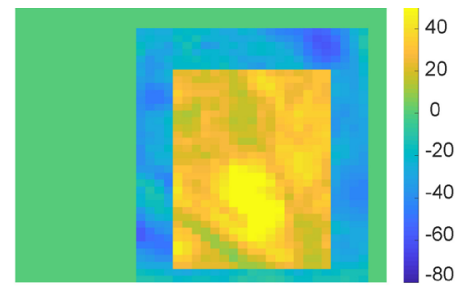

(d) Ternary RoIMasking

Fig. 4 Output feature maps of two different types of RoIMasking layer. (a) Input image. (b) Before RoIMasking, all values in the feature map are non-negative because of the ReLU layer. (c) After binary RoIMasking, regions outside the proposal are set to zero. (d) Ternary RoIMasking additionally considers a larger area, in which feature values are non-positive. 


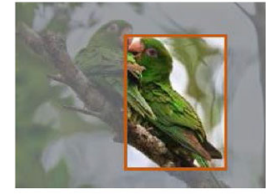

(a) Binary mask

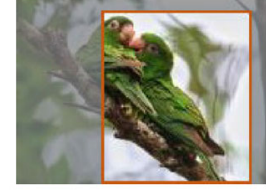

(b) Ex-Binary mask

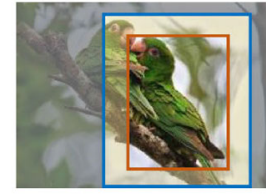

(c) Ternary mask
Fig. 5 Three different types of masks used in our RoIMasking layer (a) The binary mask only considers the regions inside the orange rectangle, (b) the expanded binary mask considers a larger rectangle than binary masking, and (c) the ternary mask takes into account both the region inside the orange rectangle and the surrounding regions marked in yellow.

branch is also single-shot. The bounding boxes predicted by the detection branch and the output of the lateral layer with stride 8 in the backbone network are fed into our segmentation branch. As shown in Fig. 2(a), our segmentation branch contains an RoIMasking layer for instance feature extraction, and a salient instance discriminator for identifying salient instances. The latter is a CNN branch used to further improve segmentation quality. The detailed structure is shown in Fig. 2(c).

\subsection{RoIMasking}

RoIPool [28] and RoIAlign [31] are standard operations for extracting fixed-size features from regions of interest. Both RoIPool and RoIAlign sample a region of interest at a fixed spatial extent of $H \times W$, typically with $H=W$, e.g., $7 \times 7$ in Ref. [28] and $28 \times 28$ in Ref. [31]. RoIPool first quantizes RoIs by uniformly dividing them into $H \times W$ spatial bins. After max-pooling each spatial bin, output feature maps with size $H \times W$ are generated. Since quantization in RoIPool is performed by rounding, it introduces misalignments between the RoI and the extracted features. As a remedy, RoIAlign avoids quantization by using bilinear interpolation.

However, both RoIPool and RoIAlign focus on regions inside the proposals, neglecting the remaining region. As discussed in Section 3.1, the region surrounding the current object RoI contains valuable information for distinguishing the target object from its background.

Unfortunately, although some layer-fusion techniques such as feature pyramid networks [32] attempt to embed comprehensive high-level information in a feature map, neither RoIPool nor RoIAlign explicitly and effectively explore the information surrounding the RoI. Moreover, the sampling process in these two operations makes them incapable of maintaining the aspect ratio and resolution of the regions of interest, possibly lowering the quality of the results. In this subsection, we design a new resolution-preserving, quantization-free layer, called RoIMasking, to take the place of RoIPool or RoIAlign. We also explore feature separation between foreground and background for improving segmentation quality.

\subsubsection{Binary RoIMasking}

We first introduce a simplified version of RoIMasking which we call binary RoIMasking. This receives feature maps and proposals predicted by the detection branch. A binary mask is generated according to the position and size of a given rectangle proposal. Values inside the rectangle are set to 1 , and outside, 0. Figure 5(a) illustrates a binary RoIMasking, in which the bright and dark areas have labels 1 and 0 , respectively. The output of the binary RoIMasking layer comprises the input feature maps multiplied by this mask. In Fig. 4, we show a typical example of the output feature maps. Unlike RoIPool [28] and RoIAlign [31], our binary RoIMasking keeps the aspect ratio and resolution of the original feature maps. In Section 4, we experimentally verify that binary RoIMasking outperforms the RoIPool and RoIAlign baselines.

\subsubsection{Expanded binary RoIMasking}

We also consider an extended version of binary RoIMasking which simply enlarges the proposed region as illustrated in Fig. 5(b). Compared to standard binary RoIMasking, expanded binary RoIMasking takes into account additional background context information, so that the segmentation branch has a larger receptive field. We will provide quantitative comparisons in our experiments section.

\subsubsection{Ternary RoIMasking}

To make better use of the background information around the regions of interest, we further extend expanded binary RoIMasking to ternary output. Because of the ReLU activation function, there are no negative values in the feature maps before RoIMasking. To explicitly notify the segmentation branch that the region outside the proposals should be considered as background, we flip the signs (i.e., set the corresponding mask values to -1 ) of feature values around the region of interest, as illustrated in yellow in Fig. 5(c). In this way, features around regions of interest are distinguished from those inside 
the bounding boxes of the salient instances. This allows the segmentation branch to be able to not only make use of features inside the region of interest as well as the surrounding context (as in extended binary RoIMasking), but also explicitly emphasises foreground-background feature separation. A feature map after ternary RoIMasking is illustrated in Fig. 4(d). Note that this operation introduces no additional computational cost into our model. Ternary RoIMasking leads to a large improvement as we show in our experiments (Section 4). In the following, for simplicity, RoIMasking refers to ternary RoIMasking unless otherwise stated.

\subsection{Analysis of RoIMasking}

We now demonstrate the importance of the background information around the regions of interest in the feature maps and the effectiveness of ternary RoIMasking. To do so, we explore the impact of each activation in the feature maps before RoIMasking on the performance. Inspired by Ref. [59], we visualize the function of a specific neuron in this model by drawing a gradient map. After loading the fully trained model weights, we do a forward pass using a specific image. In this process, the activation value of the feature maps before RoIMasking, $H_{i, j, c}$, is extracted and stored. Next, we do a backward pass. Note that in the general training stage, backpropagation is performed to calculate the gradients of the total loss with respect to the weights in the neural network, but in this experiment, we load the stored $H_{i, j, c}$ as a variable, and regard the convolution kernels as constant. Back-propagation is performed to calculate the gradients of the instance segmentation loss with respect to each feature map input to RoIMasking, i.e., $G_{i, j, c}=\partial L_{\text {sal }} / \partial H_{i, j, c}$. The absolute value of $G_{i, j, c}$ reflects the importance of the feature map pixel $H_{i, j, c}$ to the saliency task. After summing $\left|G_{i, j, c}\right|$ in the channel dimension, the gradient map $G_{i, j}$ is obtained.

Figure 6 shows the gradient maps for binary RoIMasking and ternary RoIMasking, respectively. The orange rectangle is the ground truth bounding box of a salient instance. By definition, the pixels inside the orange rectangle in the ternary mask are set to 0 and the pixels between the orange and blue boxes are set to -1 . It is obvious that there are evident responses in the background (marked as " -1 " in the ternary mask) in Fig. 6(b). In Fig. 6(a), there

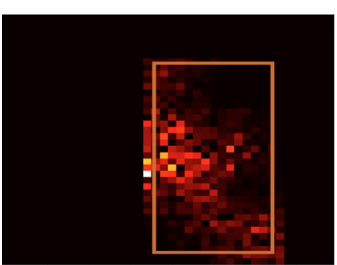

(a) Binary masking

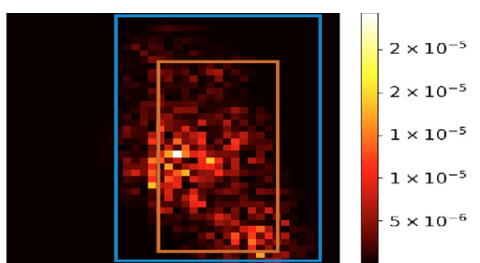

(b) Ternary masking
Fig. 6 Gradient maps using binary masking and ternary masking. Ternary masking considers more peripheral information about the region around the proposal. The input image is shown in Fig. 4(a).

are only a few responses between the orange and blue boxes. This indirectly indicates the importance of the contextual information around the regions of interest. More experimental results can be found in the experiments section.

\subsection{Segmentation branch}

Taking into account the structure of our backbone, we take the feature maps from the lateral layer associated with conv3 with a stride of 8 as the input to our segmentation branch, trading-off between global context and details. Before connecting our RoIMasking layer, we first add a simple convolutional layer with 256 channels and kernel size $1 \times 1$ to compress the number of channels. Despite the RoIMasking layer, it is still difficult to distinguish salient instances from other instances inside the same RoI. To do so, we add a segmentation branch similar to Mask-RCNN [31] to help better distinguish the instances.

As pointed out in Ref. [60], enlarging the receptive field is helpful for segmentation related tasks. Inspired by Refs. [31, 60], we design a new segmentation branch by introducing skip connections and dilated convolutional layers (see Fig. 2(c)). As well as two residual blocks, we also add two $3 \times 3 \max$ pooling layers with stride 1 and dilated convolutional layers with dilation rate 2 to enlarge the receptive field. All convolutional layers have kernel size $3 \times 3$ and stride 1 . The first three have 128 channels and the remainder, 64, which suffice for salient instance segmentation.

\subsection{Loss function}

For saliency detection, we have a human annotated ground truth saliency map. The target for the loss function is to constrain our model to predict a map for each salient object as similar as possible to the ground truth saliency map. As described above, there are two 
branches in our framework for detection and saliency segmentation, respectively. The detection branch undertakes objectness classification and coordinates the regression task, while the segmentation branch performs saliency segmentation. Therefore, we use a multi-task loss $L$ on each training sample to jointly train the model:

$$
L=L_{\mathrm{obj}}+L_{\text {coord }}+L_{\mathrm{seg}}
$$

Here, the losses are $L_{\mathrm{obj}}$ for the objectness classification task, $L_{\text {coord }}$ for the regression task, and $L_{\text {seg }}$ for the saliency segmentation branch. These loss functions are equally weighted. In this paper, positive object proposals are considered to be those with intersection-over-union (IoU) with the ground truth $>0.5$. As positive proposals are far fewer than negative samples in the detection branch, we adopt the following strategy. Let $P$ and $N$ be the sets of positive and negative proposals, and $N_{P}$ and $N_{N}$ be the numbers of positive and negative proposals $\left(N_{P} \ll N_{N}\right)$. Then we calculate the positive and negative objectness loss separately to avoid domination of negative gradients during training:

$$
L_{\mathrm{obj}}=-\left(\frac{1}{N_{P}} \sum_{i \in P} \log p_{i}+\frac{1}{N_{N}} \sum_{j \in N} \log \left(1-p_{j}\right)\right)
$$

where $p_{i}$ is the probability of the $i$ th proposal being positive.

We use Smooth $_{L 1}$ loss as in Fast-RCNN [28] for coordinate regression and cross-entropy loss as in Mask-RCNN [31] for the segmentation branch.

\section{Experiments}

In this section, we carry out a detailed analysis to evaluate the contribution of each component of our method by ablation studies. We also perform thorough comparisons with state-of-the-art methods to exhibit the effectiveness of our approach. We use the dataset proposed in Ref. [9] for all experiments. It contains 1000 images with well-annotated instancelevel annotations. For fair comparisons, as in Ref. [9], we randomly select 500 images for training, 200 for validation, and 300 for testing.

\subsection{Implementation details}

\subsubsection{Training and testing}

During training, the IoU is used to determine whether a bounding box proposal is a positive or negative sample in the detection branch. A bounding box proposal is positive if its $\mathrm{IoU}>0.5$, and negative if $\mathrm{IoU}<0.5$

In the testing phase, the bounding boxes fed into the RoIMasking layer come from the detection branch, but in the training phase, we directly feed the ground truth bounding boxes into the RoIMasking layer. This provides the segmentation branch with more stable and valid training data and meanwhile accelerates the training process, as verified by empirical experiments.

\subsubsection{Hyper-parameters}

Our proposed network is based on the TensorFlow library [61]. The input images are augmented by horizontal flipping. The hyper-parameters are set as follows: weight decay $=0.0001$, momentum $=0.9$. We train our network on 2 GPUs for 20k iterations, with an initial learning rate of 0.004 which is divided by a factor of 10 after $10 \mathrm{k}$ iterations. It only takes 40 minutes to train the whole model.

\subsection{Ablation studies}

To evaluate the effectiveness of each component in our proposed framework, we trained our model on a salient instance segmentation dataset [9]. Following standard COCO metrics [62], we report results in terms of mAP (averaged precision over IoU thresholds), including $\mathrm{mAP}^{0.5}$ and $\mathrm{mAP}^{0.7}$. Furthermore, to analyze the ability to distinguish different instances, we also consider another set which only contains instances with occlusion, which is denoted by $\mathrm{mAP}_{O}$.

\subsubsection{Effect of RoIMasking}

To evaluate the performance of the proposed RoIMasking layer, we also consider using RoIPool [28] and RoIAlign [31]. We replace our RoIMasking with RoIPool and RoIAlign in two comparative experiments while keeping all other network structures and experimental settings unchanged. Quantitative results are listed in Table 1. As can be seen, our proposed binary RoIMasking and ternary RoIMasking both outperform RoIPool and RoIAlign at $\mathrm{mAP}^{0.7}$. Specifically, ternary RoIMasking improves upon RoIAlign by around $2.1 \%$ : considering more contextual information outside the proposals helps salient instance segmentation.

To further verify the effectiveness of our RoIMasking layer, we also consider a binary masking 


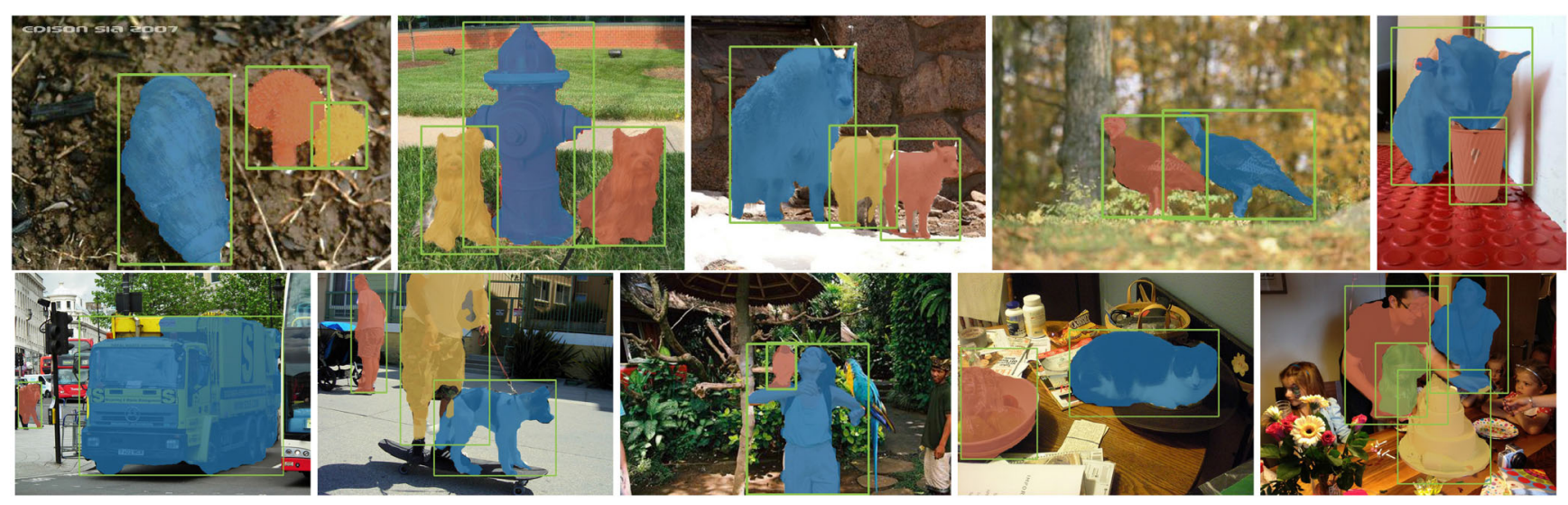

Fig. 7 Above: selected examples of instance-level saliency segmentation results on the dataset proposed by Ref. [9]. Below: examples with more complicated targets and backgrounds. Even partially occluded instances can be well distinguished and segmented by S4Net.

case in which the values in the yellow area of Fig. $5(\mathrm{~d})$ are all set to 1 . The penultimate line in Table 1 shows the corresponding results. As can be seen, the results are even worse when binary masking is used: simply enlarging the regions of interest does not help to discriminate salient instances. However, when the signs of the extended regions in the mask are flipped (ternary RoIMasking), the best results are obtained (bottom line of Table 1). This demonstrates that changing the signs of the extended regions in the mask explicitly increases the contrast between the salient instances and background. More importantly, non-salient regions inside the proposals tend to be predicted to the same class as the extended regions, so the ability to separate features between target objects and their nearby background plays an important role in our approach.

Table 1 Ablation experiments analyzing our RoIMasking layer. We also give results using RoIAlign and RoIPool proposed in Mask RCNN [31] and Fast R-CNN [28] respectively. RoIMasking outperforms RoIAlign and RoIPool even for images with occlusion

\begin{tabular}{lcccc}
\hline \multicolumn{1}{c}{ Method } & $\mathrm{mAP}^{0.5}$ & $\mathrm{mAP}^{0.7}$ & $\mathrm{mAP}_{O}^{0.5}$ & $\mathrm{mAP}_{O}^{0.7}$ \\
\hline RoIAlign & $85.2 \%$ & $61.5 \%$ & $79.2 \%$ & $47.7 \%$ \\
RoIPool & $85.2 \%$ & $61.1 \%$ & $80.3 \%$ & $50.9 \%$ \\
\hline Binary RoIMasking & $85.5 \%$ & $62.4 \%$ & $80.1 \%$ & $49.4 \%$ \\
Ternary RoIMasking & $86.7 \%$ & $63.6 \%$ & $81.2 \%$ & $51.5 \%$ \\
\hline
\end{tabular}

Table 2 Performance of S4Net with different expanded areas. All the results are based on ResNet-50 [57]. $\alpha=1 / 3$ gives the best result

\begin{tabular}{ccccccc}
\hline$\alpha$ & 0 & $1 / 6$ & $1 / 3$ & $1 / 2$ & $2 / 3$ & 1 \\
\hline $\mathrm{mAP}^{0.5}$ & $85.9 \%$ & $86.4 \%$ & $\mathbf{8 6 . 7} \%$ & $86.5 \%$ & $86.2 \%$ & $85.9 \%$ \\
$\mathrm{mAP}^{0.7}$ & $62.5 \%$ & $63.4 \%$ & $\mathbf{6 3 . 6} \%$ & $63.3 \%$ & $62.4 \%$ & $62.0 \%$ \\
\hline
\end{tabular}

\subsubsection{Size of contextual regions}

To better understand our RoIMasking layer, we analyze how large the contextual regions should be. Suppose the bounding box of a salient instance has width and height $w$ and $h$ respectively. We define an expansion coefficient $\alpha$ to denote the width of the " -1 " region in the RoI mask, so the valid region has width and height $w+2 \alpha w, h+2 \alpha h$. By default, we set $\alpha$ to $1 / 3$. We also considered various values of $\alpha$ to explore its influence on the final results as shown in Table 2, but found both larger and smaller values of $\alpha$ slightly reduce performance. This indicates that a region of this size suffices to discriminating different instances, and a larger " -1 " region may cause more salient instances to be viewed, weakening the ability to identify the "real" salient instances.

\subsubsection{Number of proposals}

The number of proposals sent to the segmentation branch also affects performance: more proposals lead to better performance but at greater computational costs. Figure 8 shows the relationship between performance and speed with increasing number of proposals.

Further performance gain is not obvious when the number of proposals exceeds 20. Specifically, when we set the number of proposals to 100, only around $1.5 \%$ improvement is achieved but the runtime cost increases dramatically. Taking this into account, we take 20 proposals as a trade-off during the inference phase. Users may tune the number of proposals according to their task. 


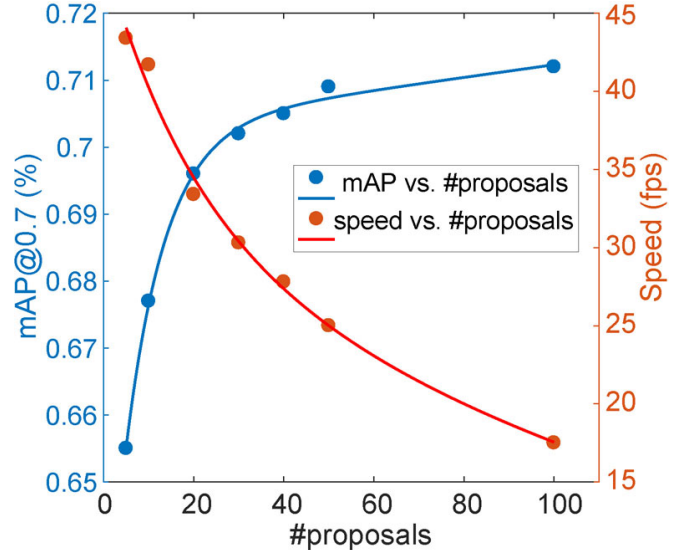

Fig. 8 With increasing proposals, S4Net performs better, but the time taken increases in parallel.

\subsubsection{Coefficients in the loss function}

S4Net requires only a few hyperparameters. Besides the expansion coefficient $\alpha$ and the number of proposals in the segmentation branch, we also analyze how the coefficients in the loss function affect the overall performance, by changing the weights for $L_{\mathrm{obj}}$ and $L_{\text {seg }}$ and retraining the model. Table 3 shows the results, indicating that $(0.3,1)$ for $\left(L_{\mathrm{obj}}, L_{\mathrm{seg}}\right)$ is the best choice; S4Net is insensitive to these coefficients when they are in a reasonable range, and tuning them brings little benefit.

\subsubsection{Base models}

Besides using ResNet-50 [57] as a base model, we also tried three other popular base models: Resnet101 [57], VGG16 [64], and MobileNet [63]. Table 4

Table 3 Performance of S4Net evaluated by $\mathrm{mAP}^{0.7}$ for different coefficients for $L_{\mathrm{obj}}$ and $L_{\mathrm{seg}}$ in the loss function, to determine the optimal settings and analyze sensitivity to these coefficients. "diverge" indicates the loss function fails to converge in training. Changing the coefficients has little impact

\begin{tabular}{cccccc}
\hline Coefficients & 0.1 & 0.3 & 1 & 3 & 10 \\
\hline$L_{\text {obj }}$ & $63.1 \%$ & $\mathbf{6 3 . 6} \%$ & $63.2 \%$ & $62.4 \%$ & $62.7 \%$ \\
$L_{\text {seg }}$ & $63.4 \%$ & $63.3 \%$ & $\mathbf{6 3 . 6} \%$ & $62.9 \%$ & diverge \\
\hline
\end{tabular}

Table 4 Performance of S4Net when using different base models When we change the default ResNet-50 to ResNet-101, $3.2 \%$ improvement is obtained at the cost of a little extra time. Using MobileNet [63] as our base model yields a frame rate of more than 90 fps on a GTX 1080 Ti GPU

\begin{tabular}{lccc}
\hline \multicolumn{1}{c}{ Base models } & $\mathrm{mAP}^{0.5}$ & $\mathrm{mAP}^{0.7}$ & Speed (fps) \\
\hline ResNet-101 [57] & $\mathbf{8 8 . 1} \%$ & $\mathbf{6 6 . 8} \%$ & 33.3 \\
ResNet-50 [57] & $86.7 \%$ & $63.6 \%$ & 40.0 \\
\hline VGG16 [64] & $82.2 \%$ & $53.0 \%$ & 43.5 \\
MobileNet [63] & $62.9 \%$ & $33.5 \%$ & 90.9 \\
\hline
\end{tabular}

lists the results using different base models. As one can see, base models with better performance on classification also work better in our experiments. For speed, real-time processing can be achieved by our proposed S4Net. When the size of input images is $320 \times 320$, S4Net has a frame rate of $40 \mathrm{fps}$ on a GTX 1080 Ti GPU. Using MobileNet [63] as our base model allows S4Net to run very fast, at a speed of 91 fps.

\subsection{Comparison with state-of-the-art}

Unlike salient object detection, which has been studied for many years, salient instance detection is a relatively new problem. There is only one related approach, MSRNet [9], that can be used for direct comparison. In this experiment, we compare our S4Net based on ResNet-50 [57] with the MSRNet method. We report the results using $\mathrm{mAP}^{0.5}$ and $\mathrm{mAP}^{0.7}$ metrics.

\subsubsection{Quantitative analysis}

Two datasets are used in our experiments. The results of comparative experiments on the dataset proposed by Ref. [9] are listed in Table 5. Our proposed S4Net achieves better results for both $\mathrm{mAP}^{0.5}$ and $\mathrm{mAP}^{0.7}$ than MSRNet [9]. Specifically, our approach improves the baseline results presented in MSRNet [9] by about 21 points in $\mathrm{mAP}^{0.5}$. We also have a great improvement on the same dataset for $\mathrm{mAP}^{0.7}$.

\subsubsection{SOC benchmark}

To further verify the the performance of our proposed framework, we also conducted an experiment on another instance-level saliency detection dataset, the SOC benchmark proposed by Ref. [65], which contains 6000 training samples and 1200 test samples. As shown in Table 6, S4Net achieves reasonable instancelevel saliency detection accuracy. Because SOC is a new benchmark, there is no public baseline for comparison, so here we can only show our own results.

Table 5 Quantitative comparison with existing methods. Instance segmentation maps of Ref. [9] and related code are unavailable, so denoted by "_-"

\begin{tabular}{ccccc}
\hline \multicolumn{1}{c}{ Methods } & $\mathrm{mAP}^{0.5}$ & $\mathrm{mAP}^{0.7}$ & $\mathrm{mAP}_{O}^{0.5}$ & $\mathrm{mAP}_{O}^{0.7}$ \\
\hline MSRNet [9] & $65.3 \%$ & $52.3 \%$ & - & - \\
S4Net & $\mathbf{8 6 . 7} \%$ & $\mathbf{6 3 . 6} \%$ & $81.2 \%$ & $51.5 \%$ \\
\hline \multicolumn{2}{c}{ Table 6 } & Saliency detection results on SOC dataset \\
\hline Method & S_measure & F_measure \\
\hline S4Net & $\mathbf{8 5 . 9} \%$ & $\mathbf{8 2 . 1} \%$ \\
\hline
\end{tabular}




\section{Applications}

In this section, we apply our proposed S4Net to a popular vision task - weakly-supervised semantic segmentation. Recent work $[33,34]$ has demonstrated that salient object detectors $[15,16,19,66]$ provide good initial heuristic cues for learning semantic segmentation. Given a collection of simple images $[33,34]$, each of which is associated with one semantic class, saliency maps offer higher quality heuristic cues than attention maps provided by attention models $[67,68]$. Despite this, modern saliency detectors often lack the ability to distinguish two adjacent salient instances. What is worse is that sometimes saliency detectors output not only semantic objects but also part of the background, causing interference when learning semantic segmentations. Fortunately, our salient instance detector is able to avoid this issue, as our method produces results with instance-level information, allowing the discarding of unimportant instances. For training samples with multiple keywords, such as the images in PASCAL VOC [36], discriminating different instances is essential for keyword assignment.

\subsection{Comparisons with related work}

To better highlight our strengths, we follow the idea proposed in Ref. [33], which presented a simple-to-complex framework for learning semantic segmentation with the support of only salient object detectors. As the goal of this section is to exhibit the superiority of our approach to regular state-ofthe-art saliency detectors, we only show the results from the first-round iteration in Ref. [33] in terms of mean IoU. Here we take the simple dataset [34] as the training set, which contains nearly 24,000 samples with one keyword per image. To generate heuristic cues for learning semantic segmentation, we refer to the instance segmentations as a binary map for each image; all the instance segmentations share the same category as the image. After predicting instance-level saliency masks for each image, we take the input keyword as category information, so complete training data with pixel-level annotation is generated. Following Ref. [33], we adopt the widelyused DeepLab-Large-FOV architecture [69] as our network. The results are shown in Table 7 . It is obvious that training with our instance segmentations on the same dataset works much better than using
Table 7 Semantic segmentation results with different initial heuristic cues on the PASCAL VOC validation set. Training with our instancelevel saliency cues greatly outperforms settings with regular saliency cues

\begin{tabular}{lll}
\hline \multicolumn{1}{c}{ Model } & \multicolumn{1}{c}{ Heuristic cues } & Val set \\
\hline DeepLab-VGG16 & Saliency maps [16] & $49.8 \%$ \\
DeepLab-VGG16 & Saliency maps [19] & $52.6 \%$ \\
DeepLab-VGG16 & Attention [70] + Saliency [19] & $53.8 \%$ \\
\hline DeepLab-VGG16 & Salient instances & $57.4 \%$ \\
DeepLab-ResNet101 & Salient instances & $\mathbf{6 1 . 8} \%$ \\
\hline
\end{tabular}

other heuristic cues. Our approach obtains a $4.8 \%$ performance gain compared to using the DSS salient object detector [19].

\subsection{Discussion}

This paper has shown the strength of replacing saliency maps by a recent state-of-the-art method [19] with our instance-level saliency maps. In fact, with the salient instance segmentations provided, we can conduct a series of high-level operations to select reliable salient instances instead of them all. For example, we can use clustering methods to group similar instances while discarding those instances far from the clustering center in some feature space for each category. The salient instances can also be combined with attention models for filtering instances with small areas detected. In summary, we wish to stress the wide range of uses of our salient instance detector when applied to weakly-supervised semantic segmentation; giving more details lies beyond the scope of this paper.

\section{Conclusions}

This paper has presented a single stage salientinstance segmentation framework, which is able to segment instance-level salient objects in real time. The key novelties include (i) the ROIMasking layer, which takes into account both information inside the proposals and contextual information outside the proposals, while preserving the original resolution and aspect ratio of regions of interest, and (2) an advanced salient instance discriminator which enlarges the receptive field of our segmentation branch, boosting performance. Thorough experiments show that the proposed RoIMasking greatly outperforms RoIAlign and RoIPool, especially for distinguishing instances in the same scope. Our S4Net achieves state-of-the-art performance on a publicly available benchmark. 


\section{Acknowledgements}

This research was supported by National Natural Science Foundation of China (61521002, 61572264, 61620106008), the National Youth Talent Support Program, Tianjin Natural Science Foundation (17JCJQJC43700, 18ZXZNGX00110), and the Fundamental Research Funds for the Central Universities (Nankai University, No. 63191501).

\section{References}

[1] Li, F. F.; VanRullen, R.; Koch, C.; Perona, P. Rapid natural scene categorization in the near absence of attention. Proceedings of the National Academy of Sciences of the United States of America Vol. 99, No. 14, 9596-9601, 2002.

[2] Elazary, L.; Itti, L. Interesting objects are visually salient. Journal of Vision Vol. 8, No. 3, 3, 2008.

[3] Cheng, M.-M.; Zhang, F.-L.; Mitra, N. J.; Huang, X. $\mathrm{Hu}$, S.-M. RepFinder: Finding approximately repeated scene elements for image editing. ACM Transactions on Graphics Vol. 29, No. 4, Article No. 83, 2010.

[4] Wu, H. S.; Wang, Y. S.; Feng, K. C.; Wong, T. T.; Lee, T. Y.; Heng, P. A. Resizing by symmetrysummarization. ACM Transactions on Graphics Vol. 29, No. 6, Article No. 159, 2010.

[5] Chen, T.; Cheng, M.-M.; Tan, P.; Shamir, A.; Hu, S.-M. Sketch2photo: Internet image montage. ACM Transactions on Graphics Vol. 28, No. 5, Article No. 124,2009

[6] Wu, C.; Lenz, I.; Saxena, A. Hierarchical semantic labeling for task-relevant RGB-D perception. In: Proceedings of the Robotics: Science and Systems, 2014.

[7] Borji, A.; Cheng, M.-M.; Hou, Q.; Jiang, H.; Li, J. Salient object detection: A survey. Computational Visual Media Vol. 5, No. 2, 117-150, 2019.

[8] Bylinskii, Z.; Judd, T.; Oliva, A.; Torralba, A.; Durand, F. What do different evaluation metrics tell us about saliency models? IEEE Transactions on Pattern Analysis and Machine Intelligence Vol. 41, No. 3, 740757,2019

[9] Li, G.; Xie, Y.; Lin, L.; Yu, Y. Instance-level salient object segmentation. In: Proceedings of the IEEE Conference on Computer Vision and Pattern Recognition, 2386-2395, 2017.

[10] Wolfe, J. M.; Horowitz, T. S. What attributes guide the deployment of visual attention and how do they do it? Nature Reviews Neuroscience Vol. 5, No. 6, 495-501, 2004 .
[11] Desimone, R.; Duncan, J. Neural mechanisms of selective visual attention. Annual Review of Neuroscience Vol. 18, No. 1, 193-222, 1995.

[12] Mannan, S. K.; Kennard, C.; Husain, M. The role of visual salience in directing eye movements in visual object agnosia. Current Biology Vol. 19, No. 6, R247R248, 2009.

[13] Itti, L.; Koch, C.; Niebur, E. A model of saliencybased visual attention for rapid scene analysis. IEEE Transactions on Pattern Analysis and Machine Intelligence Vol. 20, No. 11, 1254-1259, 1998.

[14] Itti, L.; Koc, C. Computational modeling of visual attention. Nature Reviews Neuroscience Vol. 2, No. 3, 194-203, 2001

[15] Cheng, M. M.; Mitra, N. J.; Huang, X. L.; Torr, P. H. S.; Hu, S. M. Global contrast based salient region detection. IEEE Transactions on Pattern Analysis and Machine Intelligence Vol. 37, No. 3, 569-582, 2015.

[16] Jiang, H. Z.; Wang, J. D.; Yuan, Z. J.; Wu, Y.; Zheng, N. N.; Li, S. P. Salient object detection: A discriminative regional feature integration approach. In: Proceedings of the IEEE Conference on Computer Vision and Pattern Recognition, 2083-2090, 2013.

[17] Zhu, W.; Liang, S.; Wei, Y.; Sun, J. Saliency optimization from robust background detection. In: Proceedings of the IEEE Conference on Computer Vision and Pattern Recognition, 2814-2821, 2014.

[18] Rother, C.; Kolmogorov, V.; Blake A. "GrabCut": Interactive foreground extraction using iterated graph cuts. ACM Transactions on Graphics Vol. 23, No. 3, 309-314, 2004.

[19] Hou, Q.; Cheng, M.-M.; Hu, X.; Borji, A.; Tu, Z.; Torr, P. H. S. Deeply supervised salient object detection with short connections. IEEE Transactions on Pattern Analysis and Machine Intelligence Vol. 41, No. 4, 815828, 2019

[20] Li, G.; Yu, Y. Deep contrast learning for salient object detection. In: Proceedings of the IEEE Conference on Computer Vision and Pattern Recognition, 478-487, 2016.

[21] Wang, L.; Lu, H.; Ruan, X.; Yang, M.-H. Deep networks for saliency detection via local estimation and global search. In: Proceedings of the IEEE Conference on Computer Vision and Pattern Recognition, 31833192, 2015.

[22] Dai, J.; He, K.; Sun, J. Convolutional feature masking for joint object and stuff segmentation. In: Proceedings of the IEEE Conference on Computer Vision and Pattern Recognition, 3992-4000, 2015. 
[23] Hariharan, B.; Arbeláez, P.; Girshick, R.; Malik, J. Simultaneous detection and segmentation. In: Computer Vision - ECCV 2014. Lecture Notes in Computer Science, Vol. 8695. Fleet, D.; Pajdla, T.; Schiele, B.; Tuytelaars, T. Eds. Springer Cham, 297-312, 2014.

[24] Hariharan, B.; Arbelaez, P.; Girshick, R.; Malik, J. Hypercolumns for object segmentation and fine-grained localization. In: Proceedings of the IEEE Conference on Computer Vision and Pattern Recognition, 447-456, 2015.

[25] Girshick, R.; Donahue, J.; Darrell, T.; Malik, J. Rich feature hierarchies for accurate object detection and semantic segmentation. In: Proceedings of the IEEE Conference on Computer Vision and Pattern Recognition, 580-587, 2014.

[26] Ren, S. Q.; He, K. M.; Girshick, R.; Sun, J. Faster R-CNN: Towards real-time object detection with region proposal networks. IEEE Transactions on Pattern Analysis and Machine Intelligence Vol. 39, No. 6, 11371149, 2017.

[27] Dai, J.; Li, Y.; He, K.; Sun, J. R-FCN: Object detection via region-based fully convolutional networks. In: Proceedings of the Advances in Neural Information Processing Systems 29, 2016.

[28] Girshick, R. Fast R-CNN. In: Proceedings of the IEEE International Conference on Computer Vision, 14401448, 2015.

[29] He, K. M.; Zhang, X. Y.; Ren, S. Q.; Sun, J. Spatial pyramid pooling in deep convolutional networks for visual recognition. IEEE Transactions on Pattern Analysis and Machine Intelligence Vol. 37, No. 9, 19041916, 2015.

[30] Dai, J. F.; He, K. M.; Li, Y.; Ren, S. Q.; Sun, J. Instance-sensitive fully convolutional networks. In: Computer Vision - ECCV 2016. Lecture Notes in Computer Science, Vol. 9910. Leibe, B.; Matas, J.; Sebe, N.; Welling, M. Eds. Springer Cham, 534-549, 2016.

[31] He, K.; Gkioxari, G.; Dollár, P.; Girshick, R. Mask R-CNN. In: Proceedings of the IEEE International Conference on Computer Vision, 2961-2969, 2017.

[32] Lin, T.-Y.; Dollár, P.; Girshick, R. B.; He, K.; Hariharan, B.; Belongie, S. J. Feature pyramid networks for object detection. In: Proceedings of the IEEE Conference on Computer Vision and Pattern Recognition, 2117-2125, 2017.

[33] Wei, Y. C.; Liang, X. D.; Chen, Y. P.; Shen, X. H.; Cheng, M. M.; Feng, J. S.; Zhao, Y.; Yan, S. STC: A simple to complex framework for weakly-supervised semantic segmentation. IEEE Transactions on Pattern Analysis and Machine Intelligence Vol. 39, No. 11, 23142320, 2017.
[34] Hou, Q. B.; Massiceti, D.; Dokania, P. K.; Wei, Y. C.; Cheng, M. M.; Torr, P. H. S. Bottom-up top-down cues for weakly-supervised semantic segmentation. In: Energy Minimization Methods in Computer Vision and Pattern Recognition. Lecture Notes in Computer Science, Vol. 10746. Pelillo, M.; Hancock, E. Eds. Springer Cham, 263-277, 2018.

[35] Russakovsky, O.; Deng, J.; Su, H.; Krause, J.; Satheesh, S.; Ma, S.; Huang, Z.; Karpathy, A.; Khosla, A.; Bernstein, M. et al. ImageNet large scale visual recognition challenge International Journal of Computer Vision Vol. 115, 211-252, 2015.

[36] Everingham, M.; Eslami, S. M. A.; van Gool, L.; Williams, C. K. I.; Winn, J.; Zisserman, A. The pascal visual object classes challenge: A retrospective. International Journal of Computer Vision Vol. 111, No. 1, 98-136, 2015.

[37] Zhang, J. M.; Sclaroff, S.; Lin, Z.; Shen, X. H.; Price, B.; Mech, R. Unconstrained salient object detection via proposal subset optimization. In: Proceedings of the IEEE Conference on Computer Vision and Pattern Recognition, 5733-5742, 2016.

[38] Pont-Tuset, J.; Arbelaez, P.; Barron, J. T.; Marques, F.; Malik, J. Multiscale combinatorial grouping for image segmentation and object proposal generation. IEEE Transactions on Pattern Analysis and Machine Intelligence Vol. 39, No. 1, 128-140, 2017.

[39] Qi, W.; Cheng, M. M.; Borji, A.; Lu, H. C.; Bai, L. F. SaliencyRank: Two-stage manifold ranking for salient object detection. Computational Visual Media Vol. 1, No. 4, 309-320, 2015.

[40] Borji, A.; Cheng, M. M.; Jiang, H. Z.; Li, J. Salient object detection: A benchmark. IEEE Transactions on Image Processing Vol. 24, No. 12, 5706-5722, 2015.

[41] Achanta, R.; Shaji, A.; Smith, K.; Lucchi, A.; Fua, P.; Süsstrunk, S. SLIC superpixels compared to stateof-the-art superpixel methods. IEEE Transactions on Pattern Analysis and Machine Intelligence Vol. 34, No. 11, 2274-2282, 2012.

[42] Felzenszwalb, P. F.; Huttenlocher, D. P. Efficient graphbased image segmentation. International Journal of Computer Vision Vol. 59, No. 2, 167-181, 2004.

[43] Shi, J. B.; Malik, J. Normalized cuts and image segmentation. IEEE Transactions on Pattern Analysis and Machine Intelligence Vol. 22, No. 8, 888-905, 2000.

[44] Wang, J. D.; Jiang, H. Z.; Yuan, Z. J.; Cheng, M. M.; $\mathrm{Hu}, \mathrm{X}$. W.; Zheng, N. N. Salient object detection: A discriminative regional feature integration approach. International Journal of Computer Vision Vol. 123, No. 2, 251-268, 2017.

[45] Zhao, R.; Ouyang, W.; Li, H.; Wang, X. Saliency detection by multi-context deep learning. In: 
Proceedings of the IEEE Conference on Computer Vision and Pattern Recognition, 1265-1274, 2015.

[46] Lee, G.; Tai, Y.-W.; Kim, J. Deep saliency with encoded low level distance map and high level features. In: Proceedings of the IEEE Conference on Computer Vision and Pattern Recognition, 660-668, 2016.

[47] Li, G.; Yu, Y. Visual saliency based on multiscale deep features. In: Proceedings of the IEEE Conference on Computer Vision and Pattern Recognition, 5455-5463, 2015.

[48] Lowe, D. G. Distinctive image features from scaleinvariant keypoints. International Journal of Computer Vision Vol. 60, No. 2, 91-110, 2004.

[49] Bay, H.; Ess, A.; Tuytelaars, T.; Van Gool, L. Speededup robust features (SURF). Computer Vision and Image Understanding Vol. 110, No. 3, 346-359, 2008.

[50] Dalal N.; Triggs, B. Histograms of oriented gradients for human detection. In: Proceedings of the IEEE Computer Society Conference on Computer Vision and Pattern Recognition, Vol. 1, 886-893, 2005.

[51] Sermanet, P.; Eigen, D.; Zhang, X.; Mathieu, M.; Fergus, R.; LeCun, Y. Overfeat: Integrated recognition, localization and detection using convolutional networks. arXiv preprint arXiv:1312.6229, 2013.

[52] Uijlings, J. R.; Van De Sande, K. E.; Gevers, T.; Smeulders, A. W. Selective search for object recognition. International Journal of Computer Vision Vol. 104, No. 2, 154-171, 2013.

[53] Cheng, M.-M.; Zhang, Z.; Lin, W.-Y.; Torr, P. BING: Binarized normed gradients for objectness estimation at 300fps. In: Proceedings of the IEEE Conference on Computer Vision and Pattern Recognition, 3286-3293, 2014.

[54] Pinheiro, P. O.; Collobert, R.; Dollár, P. Learning to segment object candidates. In: Proceedings of the Advances in Neural Information Processing Systems $28,2015$.

[55] Arbeláez, P.; Maire, M.; Fowlkes, C.; Malik, J. Contour detection and hierarchical image segmentation. IEEE Transactions on Pattern Analysis and Machine Intelligence Vol. 33, No. 5, 898-916, 2011.

[56] Li, Y.; Qi, H.; Dai, J.; Ji, X.; Wei, Y. Fully convolutional instance-aware semantic segmentation. In: Proceedings of the IEEE Conference on Computer Vision and Pattern Recognition, 2359-2367, 2017.

[57] He, K.; Zhang, X.; Ren, S.; Sun, J. Deep residual learning for image recognition. In: Proceedings of the IEEE Conference on Computer Vision and Pattern Recognition, 770-778, 2016.

[58] Lin, T.-Y.; Goyal, P.; Girshick, R.; He, K.; Dollár, P. Focal loss for dense object detection. In: Proceedings of the IEEE International Conference on Computer Vision, 2980-2988, 2017.
[59] Yosinski, J.; Clune, J.; Nguyen, A.; Fuchs, T.; Lipson, H. Understanding neural networks through deep visualization. arXiv preprint arXiv:1506.06579, 2015 .

[60] Zhao, H.; Shi, J.; Qi, X.; Wang, X.; Jia, J. Pyramid scene parsing network. In: Proceedings of the IEEE Conference on Computer Vision and Pattern Recognition, 2881-2890, 2017.

[61] Abadi, M.; Agarwal, A.; Barham, P.; Brevdo, E.; Chen, Z.; Citro, C.; Corrado, G. S.; Davis, A.; Dean, J.; Devin, M. et al. Tensorflow: Large-scale machine learning on heterogeneous distributed systems. arXiv preprint arXiv:1603.04467, 2016.

[62] Lin, T. Y.; Maire, M.; Belongie, S.; Hays, J.; Perona, P.; Ramanan, D.; Dollár, P.; Zitnick, C. L. Microsoft COCO: Common objects in context. In: Computer Vision - ECCV 2014. Lecture Notes in Computer Science, Vol. 8693. Fleet, D.; Pajdla, T.; Schiele, B.; Tuytelaars, T. Eds. Springer Cham, 740-755, 2014.

[63] Howard, A. G.; Zhu, M.; Chen, B.; Kalenichenko, D.; Wang, W.; Weyand, T.; Andreetto, M.; Adam, H. Mobilenets: Efficient convolutional neural networks for mobile vision applications. arXiv preprint arXiv:1704.04861, 2017.

[64] Simonyan, K.; Zisserman, A. Very deep convolutional networks for large-scale image recognition. arXiv preprint arXiv:1409.1556, 2014.

[65] Fan, D. P.; Cheng, M. M.; Liu, J. J.; Gao, S. H.; Hou, Q. B.; Borji, A. Salient objects in clutter: Bringing salient object detection to the foreground. In: Computer Vision - ECCV 2018. Lecture Notes in Computer Science, Vol. 11219. Ferrari, V.; Hebert, M.; Sminchisescu, C.; Weiss, Y. Eds. Springer Cham, 196-212, 2018.

[66] Liu, N.; Han, J. DHSNet: Deep hierarchical saliency network for salient object detection. In: Proceedings of the IEEE Conference on Computer Vision and Pattern Recognition, 678-686, 2016.

[67] Kolesnikov, A.; Lampert, C. H. Seed, expand and constrain: Three principles for weakly-supervised image segmentation. In: Computer Vision - ECCV 2016. Lecture Notes in Computer Science, Vol. 9908. Leibe, B.; Matas, J.; Sebe, N.; Welling, M. Eds. Springer Cham, 695-711, 2016.

[68] Wei, Y. C.; Feng, J. S.; Liang, X. D.; Cheng, M. M.; Zhao, Y.; Yan, S. C. Object region mining with adversarial erasing: A simple classification to semantic segmentation approach. In: Proceedings of the IEEE Conference on Computer Vision and Pattern Recognition, 6488-6496, 2017.

[69] Chen, L. C.; Papandreou, G.; Kokkinos, I.; Murphy, K.; Yuille, A. L. DeepLab: Semantic image segmentation with deep convolutional nets, atrous convolution, and 
fully connected CRFs. IEEE Transactions on Pattern Analysis and Machine Intelligence Vol. 40, No. 4, 834$848,2018$.

[70] Zhang, J. M.; Lin, Z.; Brandt, J.; Shen, X. H.; Sclaroff, $\mathrm{S}$. Top-down neural attention by excitation backprop. In: Computer Vision - ECCV 2016. Lecture Notes in Computer Science, Vol. 9908. Leibe, B.; Matas, J.; Sebe, N.; Welling, M. Eds. Springer Cham, 543-559, 2016 .

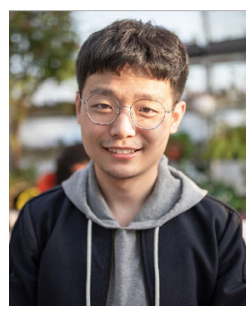

Ruochen Fan is a master student in the Computer Science Department, Tsinghua University under the supervision of Prof. Shi-Min Hu. He currently focuses on perception systems for autonomous driving, especially point cloud segmentation and RGB detection. Previously, he worked on saliency detection and weakly-supervised segmentation.

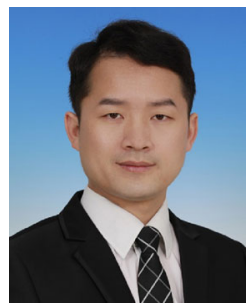

Ming-Ming Cheng is a professor in the College of Computer Science, Nankai University, leading the Media Computing Lab. He received his $\mathrm{Ph}$.D. degree from Tsinghua University in 2012. Then he worked as a research fellow for 2 years with Prof. P. Torr in Oxford. Dr. Cheng's research primarily centers on algorithmic issues in image understanding and processing, including image segmentation, editing, retrieval, etc. He has published over 30 papers in leading journals and conferences, such as IEEE TPAMI, ACM TOG, ACM SIGGRAPH, IEEE CVPR, and IEEE ICCV.

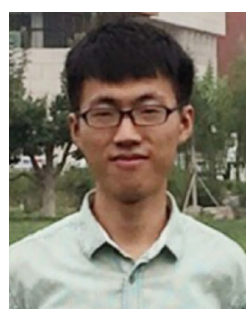

Qibin Hou is a Ph.D. student under Prof. Ming-Ming Cheng's supervision. Before joining the Media Computing Lab at Nankai University, he was a machine learning engineer in Baidu. His research interests include low-level vision, deep learning, and multimedia applications.

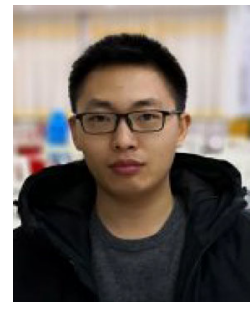

Tai-Jiang $\mathbf{M u}$ is an Assistant Researcher in the Graphics and Geometric Computing Group in the Department of Computer Science and Technology at Tsinghua University. He received his bachelor degree and Ph.D. degree in Computer Science from Tsinghua University in 2011 and 2016 respectively. His research interests are in computer graphics, image and video processing, and stereoscopic perception.

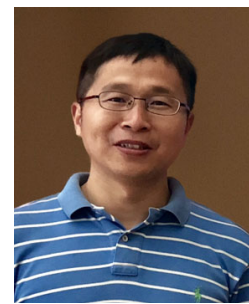

Jingdong Wang Jingdong Wang is a senior researcher in the Visual Computing Group, Microsoft Research Asia. His areas of interest include efficient CNN architecture design, human pose estimation, semantic segmentation, image classification, object detection, large-scale indexing, and salient object detection. He is serving or has served as an Associate Editor of IEEE TPAMI, IEEE TMM, and IEEE TCSVT, and an area chair (or SPC) of various prestigious conferences in vision, multimedia, and AI, such as CVPR, ICCV, ECCV, ACM MM, IJCAI, and AAAI. He is an ACM Distinguished Member and a Fellow of IAPR.

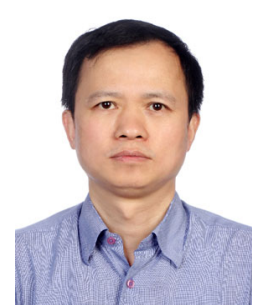

Shi-Min $\mathbf{H u}$ is a professor in the Department of Computer Science and Technology, Tsinghua University. He received his Ph.D. degree from Zhejiang University in 1996. His research interests include digital geometry processing, video processing, rendering, computer animation, and computer aided geometric design. He has published more than 100 papers in journals and refereed conferences. He is the Editor-in-Chief of Computational Visual Media, and on the editorial boards of several other journals, including Computer Aided Design and Computers \& Graphics.

Open Access This article is licensed under a Creative Commons Attribution 4.0 International License, which permits use, sharing, adaptation, distribution and reproduction in any medium or format, as long as you give appropriate credit to the original author(s) and the source, provide a link to the Creative Commons licence, and indicate if changes were made.

The images or other third party material in this article are included in the article's Creative Commons licence, unless indicated otherwise in a credit line to the material. If material is not included in the article's Creative Commons licence and your intended use is not permitted by statutory regulation or exceeds the permitted use, you will need to obtain permission directly from the copyright holder.

To view a copy of this licence, visit http:// creativecommons.org/licenses/by/4.0/.

Other papers from this open access journal are available free of charge from http://www.springer.com/journal/41095. To submit a manuscript, please go to https://www. editorialmanager.com/crmj. 